\title{
Synthesis and analysis of abnormal wurtzite ZnSe nanowheels
}

\author{
Lei Jin, ${ }^{\text {a) }}$ Wallace C. H. Choy, ${ }^{\text {b) }}$ Yee P. Leung, and Tong I. Yuk \\ Department of Electrical and Electronic Engineering, University of Hong Kong, Pokfulam Road, \\ Hong Kong, China \\ Hock C. Ong \\ Department of Physics, The Chinese University of Hong Kong, Shatin, Hong Kong, China \\ Jian-bo Wang \\ Department of Physics, Wuhan University, Wuhan 430072, China \\ and Center for Electron Microscopy, Wuhan University, Wuhan 430072, China
}

(Received 5 March 2007; accepted 30 June 2007; published online 17 August 2007)

\begin{abstract}
An abnormal structure of the ZnSe nanowheels composed of teethlike extended patterns on nanoring bases has been successfully synthesized by thermal evaporation method. It is interesting to note that the as-synthesized ZnSe nanowheels are metastable wurtzite phase with the dominant exposed surfaces of $\pm(2 \overline{1} \overline{10})$ while the stable ZnSe is typically zinc blende phase. A full picture of the growth mechanism of the metastable wurtzite phase ZnSe nanostructures will be proposed from the thermodynamic point of view. Meanwhile, the formation of the nanowheels is also explained by a two-stage mechanism. In the first stage, the base of the nanowheel begins to form by vapor-solid mechanism, while in the second stage, the teethlike extended structures grow through the self-catalyzed growth process. The cathodoluminescence spectrum of ZnSe nanowheel exhibited a band edge transition at about $460 \mathrm{~nm}$ and a strong self-activated luminescence at $610 \mathrm{~nm}$. It is important to note that the discussions of the nanostructure thermodynamics and stability can be applied to understand the growth mechanism of other nanostructures which are critical for optimization of the nanostructures. (C) 2007 American Institute of Physics.
\end{abstract}

[DOI: 10.1063/1.2769329]

\section{INTRODUCTION}

Recently, quasi-one-dimension (1D) nanostructures of the II-VI semiconductors have attracted particular interests not only for the short wavelength optical devices ${ }^{1}$ but also for photocatalysts in photochemical reaction. ${ }^{2}$ Applications of these materials for solar cells are also being studied. ${ }^{3}$ Excitons in these compound semiconductors are much more stable than those in the conventional III-V semiconductors. Recent studies showed that the low-dimensional nanostructures of Zn-based II-VI wide-gap semiconductors exhibit exciton binding energies exceeding their optical phonon energies. As a consequence, the quantum yield of the II-VI-based devices is expected to be comparable to or greater than that of the common III-V-based devices. ${ }^{4}$ Due to the potential applications in nanoelectronics, considerable efforts have been made to synthesize various morphologies of nanostructures, such as nanorods, nanowires, nanoribbons, nanobelts, ${ }^{5}$ and some advanced nanostructures. ${ }^{6,7}$ Meanwhile, the fabrication of the arrays of nanostructures has also been reported. $^{8}$

As one of the most important direct wide-band-gap II-VI group semiconductors, zinc selenide $(\mathrm{ZnSe})$ with the bulkcrystal transition energy of $2.7 \mathrm{eV}$ at $300 \mathrm{~K}$ (Ref. 9) exhibits a great potential for various optoelectronic devices ${ }^{10}$ and ap-

\footnotetext{
a) Also at the Department of Physics and Center for Electron Microscopy, Wuhan University, Wuhan 430072, China.

b) Author to whom correspondence should be addressed; electronic email: chchoy@eee.hku.hk
}

plications in spintronics, ${ }^{11}$ as well as for energy upconversion and terahertz generation in a two-step optical pumping regime. ${ }^{12} \mathrm{ZnSe}$ has two structural polymorphs of wurtzite and zinc blende. ${ }^{13} \mathrm{ZnSe}$-based nanostructures with different forms have been widely investigated recently, such as nanowires, nanoribbons, nanoneedles as well as $\mathrm{ZnSe}$ nanorods on in situ synthesized ZnSe grains. ${ }^{4,14}$ However, due to the thermodynamically unstable wurtzite phase of $\mathrm{ZnSe}$ at ambient conditions, ${ }^{13}$ most of the reported $\mathrm{ZnSe}$ nanostructures were zinc blende phase and the wurtzite structures were limitedly studied. $^{4,15}$

Here we report the fabrication of interesting ZnSe nanowheels with metastable wurtzite phase. The morphology of the as-synthesized $\mathrm{ZnSe}$ nanowheels is discussed in Sec. III A. The results show that wurtzite ZnSe nanostructures form. The formation of the as-grown nanowheels is also been proposed in Sec. III B. The detailed discussions of the growth mechanism of the nanowheels and the stability of wurtzite nanostructure are made in Sec. III C. We show that the formation of wurtzite $\mathrm{ZnSe}$ can be explained by the temperature effects and a discussion based on the thermodynamic viewpoint is also proposed to conclude the formation mechanism. Our results also show that the surface energy plays an important role in the existence of wurtzite $\mathrm{ZnSe}$ nanostructures, which may be applied for the controlled synthesis of stable wurtzite $\mathrm{ZnSe}$ nanostructures. Cathodoluminescence of $\mathrm{ZnSe}$ nanorings will be studied in Sec. III D. Finally, conclusions will be drawn in part Sec. IV. 


\section{EXPERIMENT}

The nanowheels were grown in a tube furnace. About 0.5 g high-purity ZnSe powders (99.99\% Sigma Aldrich) were put in an alumina boat and the boat was then inserted to the center region of the furnace. The entire length of the tube furnace is $100 \mathrm{~cm}$. The silicon (100) substrate, coated with about $5 \mathrm{~nm}$ of gold film, was placed $20 \mathrm{~cm}$ downstream from the $\mathrm{ZnSe}$ source. The system had then been evacuated for $30 \mathrm{~min}$ and then purged with the carrier gas of high-purity argon premixed with 5\% hydrogen at a rate of about 200 SCCM (SCCM denotes cubic centimeter per minute at $\mathrm{STP}$ ) for several minutes to remove the residual oxygen. After that, the flow rate of the carrier gas and the pressure inside the tube were maintained, respectively, at 200 SCCM and 100 torr throughout the growth process. The furnace was then heated up to $300{ }^{\circ} \mathrm{C}$ at a rate of $9{ }^{\circ} \mathrm{C} / \mathrm{min}$, then heated at a rate of $4{ }^{\circ} \mathrm{C} / \mathrm{min}$ to $950{ }^{\circ} \mathrm{C}$ and held at this temperature where the temperature was measured at the center of the furnace. Growth time in the range of 30-90 min was applied to synthesize the nanostructures. The growth temperature at the substrate increases from $\sim 650$ to $\sim 700{ }^{\circ} \mathrm{C}$ by increasing the growth time from 30 to $90 \mathrm{~min}$, respectively. After the growth, the system was cooled naturally to room temperature. Light yellowish products were obtained on the $\mathrm{Si}$ substrate after growth.

The field emission scanning electron microscopic (FESEM) images of the as-synthesized samples were taken on LEO-1530 SEM. The transmission electron microscopic (TEM) images and high resolution TEM (HRTEM) images were obtained by using JEOL-2010 (HT) and JEOL2010FEF transmission electron microscopes, respectively, both at an acceleration voltage of $200 \mathrm{kV}$. Energy-dispersive $\mathrm{X}$-ray (EDX) spectrum and the cathodoluminescence (CL) image and spectrum were recorded on the CL system attached to SEM.

\section{RESULTS AND DISCUSSION}

\section{A. Morphology of wurtzite ZnSe nanowheels}

Figure 1(a) shows the SEM image of an as-synthesized sample grown at the source temperature of $950{ }^{\circ} \mathrm{C}$ for $90 \mathrm{~min}$ in a low magnification. It can be seen that wheel-like structures with a diameter of about $40 \mu \mathrm{m}$ are obtained. From the high magnification SEM image of a typical nanowheel as shown in Fig. 1(b), there are lots of toothlike structures extended from the inner wall of nanowheels. Meanwhile, the nanowheels are very thin with a value not more than a few tens of nanometers. As a result, we can see through a nanowheel and observe the teeth patterns of another nanowheel underneath, as shown in Fig. 1(c). With a careful investigation, it is found that no particle is observed near the nanowheels. Besides, an obvious interface forms between the base and the extended parts, as shown in Fig. 1(c). The EDX spectrum as shown in Fig. 1(d) suggests that the as-synthesized nanowheels are composed of $\mathrm{Zn}$ and Se, while the Al signal comes from the SEM sample holder. Since the nanowheels are very thin, they are easily broken into pieces in the preparation of the TEM samples, and the extended toothlike structures in the inner side of nanowheels become shorter due to the same reason. Figures 2(a) and 2(b) are the TEM images of a nanowheel and the corresponding HRTEM image, respectively. Figure 2(c) is the fast Fourier transform (FFT) pattern of the base area labeled as B. It reveals that the base area of the as-synthesized nanowheel is ZnSe wurtzite structure growing along the [01 $\overline{1} 0]$ direction and the teeth pattern grown on the (0001) face of the based structure. An obvious interface can be observed again between the base and the toothlike (labeled as A) areas, which is consistent with the observation in SEM image, as shown in Fig. 1(c). The HRTEM image of the toothlike structures in Fig. 2(d) reveals that the disordered structures have mismatch dislocations and stacking faults generated along the [0001] direction.

\section{B. Formation of the nanowheels}

From the SEM image shown in Fig. 3(a), we can explain the formation of the nanowheel with teethlike extended structures using a combination of vapor-solid (VS) mechanism ${ }^{4}$ and the self-catalyzed growth process ${ }^{16}$ in two stages. Firstly, a beltlike base (see the long arrowhead) grows along $[01 \overline{1} 0]$ to form the wurtzite nanowheels via VS mechanism, with $\pm(2 \overline{1} 10)$ and $\pm(0001)$ as its top/bottom and side surfaces. In the second stage, the $\mathrm{ZnSe}$ vapor continues nucleating and grows along the [0001] direction, hence forms the extensive toothlike parts, as pointed by the short arrowheads in Fig. 3(a). When two adjacent teeth meet, strain is generated. This can be proved by the formation of mismatch dislocations in the teeth patterns, as shown in Fig. 2(d). Finally, a nanowheel with toothlike extended structures in its inner wall forms [Fig. 1(b)]. The results show that the inner side surface is (0001) plane and should be zinc terminated so that it is chemically active and could initiate growth in the direction perpendicular to the nanobelt, as reported by Wang et al. ${ }^{16}$ Meanwhile, the outer side surface of (000) $\left.\overline{1}\right)$ is smooth without any extended structures and thus should be selenium terminated. ${ }^{16}$

Schematic illustrations of the as-grown nanowheel and our early work on $\mathrm{ZnSe}$ nanoring ${ }^{17}$ are shown in Figs. 3(b) and 3(c), respectively. The growth of the $\mathrm{ZnSe}$ nanowheels and nanorings can be understood by the electrostatic polar charge model proposed by Kong and Wang. ${ }^{6}$ A dipole moment is set up due to the formation of positively charged Zn-terminate and negatively charged Se-terminate faces. In our case, the dipole moment is generated between the opposite sides of the nanobelts. In order to minimize the electrostatic energy of the entire system, the nanobelts will tend to coil up and neutralize the dipole moment by symmetric charge distribution. ${ }^{6}$ The diameter of the present nanowheels is about $40-50 \mu \mathrm{m}$ which is larger than that of our previous reported nanorings with a value of only about $10-15 \mu \mathrm{m} .{ }^{17}$ The larger diameter here can be explained by the coil thickness along the [0001] direction. According to the relationship for the change of total energy contributed by spontaneous polarization and elasticity, ${ }^{6}$ since the thickness along the [0001] direction of the nanowheels here is larger than that of 

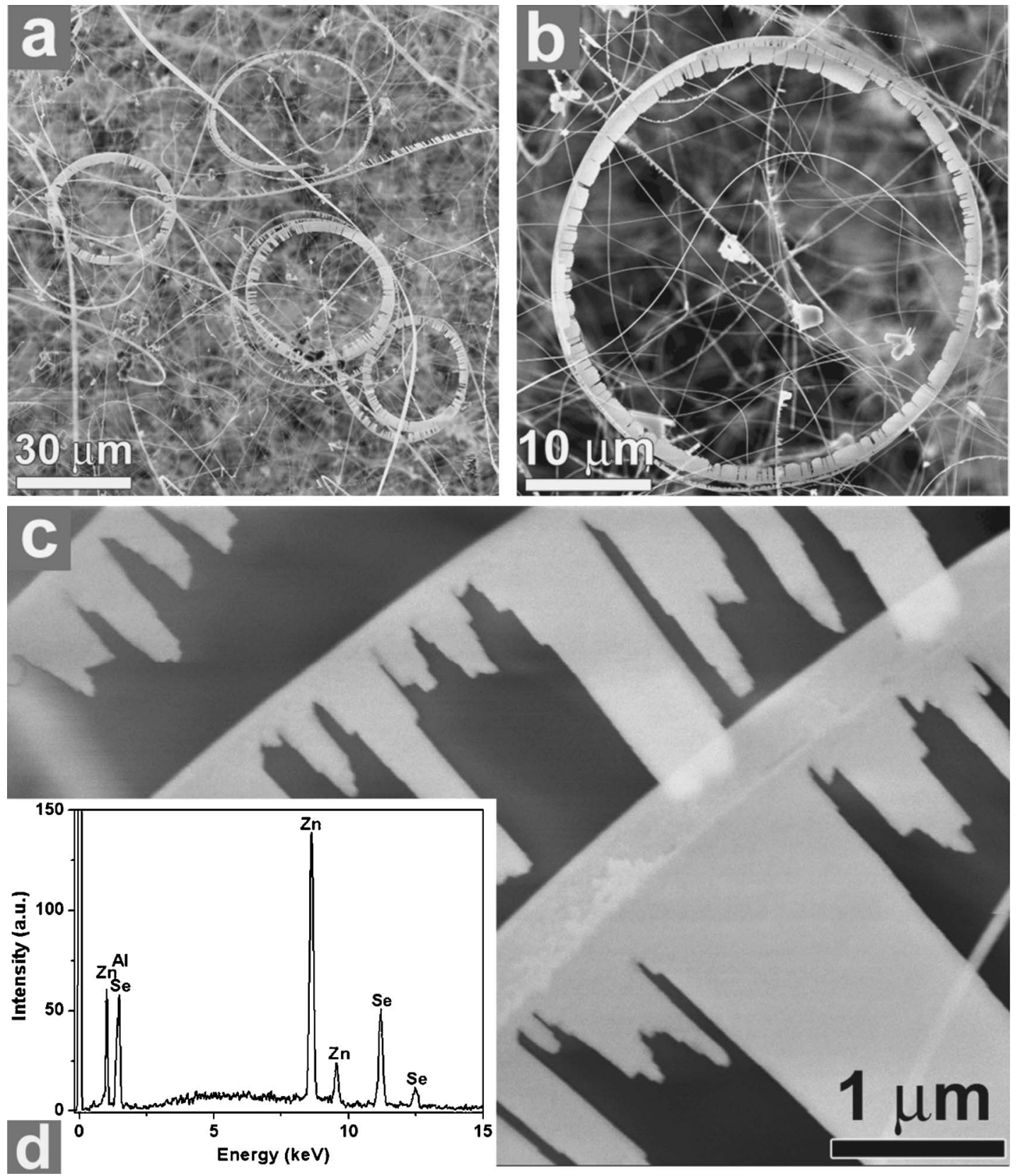

FIG. 1. SEM images and EDX spectrum of the as-synthesized sample grown at the source temperature of $950{ }^{\circ} \mathrm{C}$ for 90 min. (a) Low resolution SEM image showing the nanowheels. (b) High magnification SEM image of a typical nanowheel. (c) Enlarged image of the as-grown nanowheels with obvious interfaces between the base and the toothlike parts. (d) EDX spectrum of a nanowheel.

the previous nanorings, the diameter tends to become larger for the former case in order to minimize the change of the total electrostatic and elastic energies.

It is noted that the as-synthesized nanowheels have the dominant surfaces of $\pm(2 \overline{1} \overline{1} 0)$ planes, which is different from our previous nanoring results with the dominant surfaces of $\pm(0001)$ planes. ${ }^{17}$ Due to the dominant surfaces of $\pm(2 \overline{1} \overline{1} 0)$ faces with the lowest surface energies, ${ }^{18}$ the as-grown nanowheels should be more stable. Details will be discussed in Sec. III C.

We also found that the phase of the $\mathrm{ZnSe}$ nanostructures is sensitive to the growth temperature. By reducing the growth time from 90 to $30 \mathrm{~min}$, the growth temperature at the substrate was decreased from $\sim 700$ to $\sim 650{ }^{\circ} \mathrm{C}$. Zinc

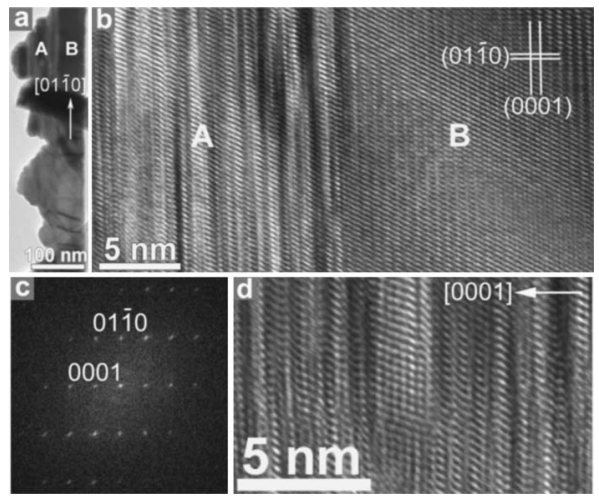

FIG. 2. (a) TEM image of the wurtzite nanowheel. (b) HRTEM image of the nanowheel showing an obvious interface. (c) FFT pattern corresponding to (b). (d) HRTEM image of the toothlike parts showing the mismatch dislocations and stacking faults. 


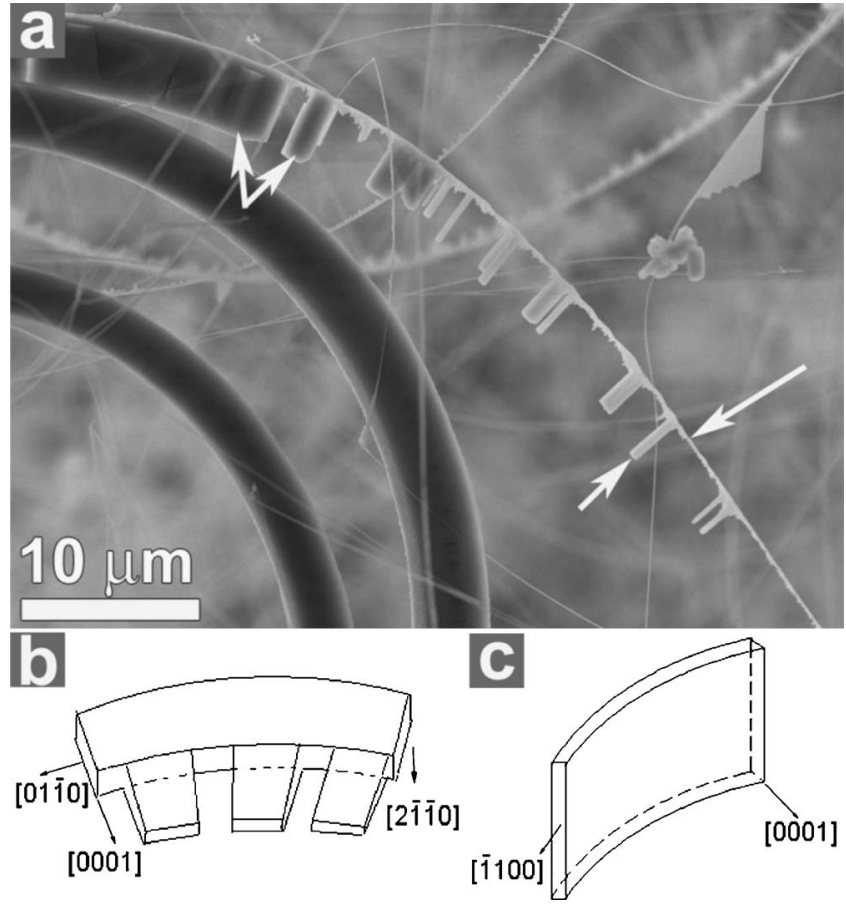

FIG. 3. (a) SEM image of wurtzite nanowheels grown at a source temperature of $950{ }^{\circ} \mathrm{C}$ for $90 \mathrm{~min}$ and 100 torr indicating the two-step growth. (b) Schematic diagram of the as-grown nanowheel. (c) Schematic diagram of the nanoring (Ref. 17) showing the [0001] direction pointing to the center of the circle.

blende structured nanowires were synthesized instead of the wurtzite nanostructures. The SEM image of the as-grown nanowires is shown in Fig. 4(a) and the inset is the magnified SEM image showing a single nanowire near its tip. The TEM image and the corresponding HRTEM image grown for

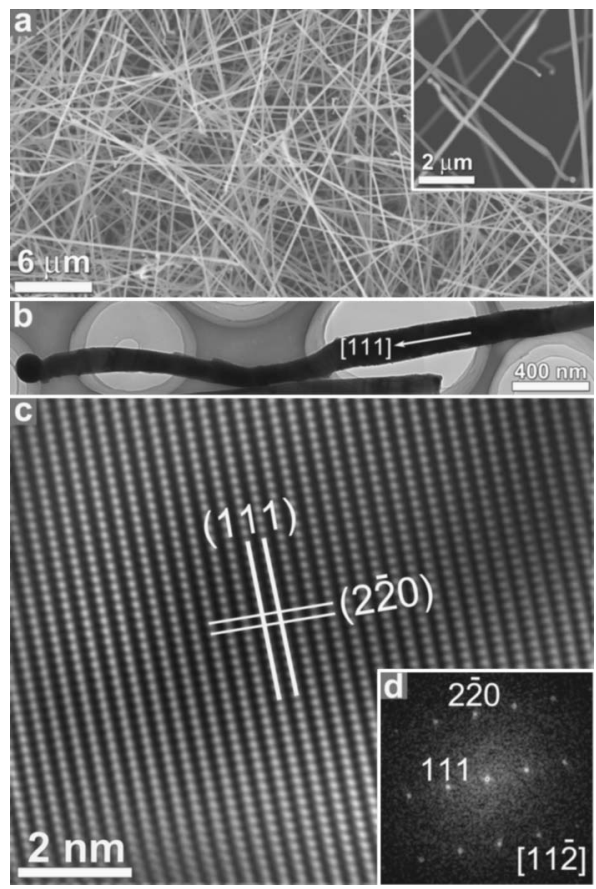

FIG. 4. (a) SEM image, (b) TEM image, (c) the corresponding HRTEM image, and (d) the FFT pattern of the nanowires grown at the source temperature of $950{ }^{\circ} \mathrm{C}$ for $30 \mathrm{~min}$. The inset of (a) is the magnified SEM image showing a single zinc blende nanowire near its tip.
30 min are shown in Figs. 4(b) and 4(c), respectively, Figure $4(d)$ is the FFT pattern. The results show that the nanowire is zinc blende $\mathrm{ZnSe}$ structure with the growth direction along [111], whereas the lateral side is along the [1피 direction. We can also find a dark particle on the tip of the nanowire, which implies that the growth of the $\mathrm{ZnSe}$ nanowire is based on the well-known metal-catalytic vapor-liquid-solid (VLS) mechanism. ${ }^{19}$ This is quite different from the result of nanowheels as shown in Fig. 2, which can be understood in terms of the VS mechanism. ${ }^{4}$ The details of zinc blende and wurtzite $\mathrm{ZnSe}$ structures synthesized at the growth times of 30 and 90 min, respectively, will be discussed in detail in Sec. III C.

\section{How to grow thermodynamically unstable wurtzite ZnSe nanostructures?}

There are a few reports on synthesizing pure wurtzite ZnSe nanostructures; ${ }^{4,15}$ yet there are no detailed discussions on the formation and stability of the wurtzite $\mathrm{ZnSe}$ nanostructures which are particularly important issues because wurtzite $\mathrm{ZnSe}$ is thermodynamically unstable at ambient conditions. We first focus on the formation mechanism of the wurtzite nanostructures and then discuss the stability of the wurtzite $\mathrm{ZnSe}$ nanostructures particularly at room temperature. As we know in bulk $\mathrm{ZnSe}$, the zinc blende structure transforms to the wurtzite structure when the temperature is above the transition temperature $\left(T_{\text {tr }}\right)$ of $1411^{\circ} \mathrm{C}$. The transformation from wurtzite structure to zinc blende structure will occur by cooling. ${ }^{20}$ However, decreasing the particle size will significantly reduce $T_{\mathrm{tr}} \cdot{ }^{21}$ According to our results, the final product changes from zinc blende structures, as shown in Fig. 4, to wurtzite structures, as shown in Fig. 2, when the growth temperature increases from 650 to $700{ }^{\circ} \mathrm{C}$ by increasing the growth time from 30 to 90 min. Meanwhile, the reactor pressure is maintained at the same value of 100 torr throughout the growth. It should be noted that the phase of crystal structure depends on the pressure and temperature of the chamber; however, the phase is not sensitive to the growth time. ${ }^{22}$ As a result, it is reasonable to consider that after $30 \mathrm{~min}$, the deposition temperature will reach or be even over $T_{\text {tr }}$ of the nanostructures. Consequently, wurtzite $\mathrm{ZnSe}$ nanostructures form. Our results are supported by the similar findings of pure wurtzite structured $\mathrm{ZnS}$ nanobelts synthesized by Ding et al. using a deposition temperature of $\sim 750{ }^{\circ} \mathrm{C}$, while $T_{\text {tr }}$ of bulk $\mathrm{ZnS}$ was reported at a higher temperature of $1020^{\circ} \mathrm{C} .^{23}$

A detailed understanding of the formation of wurtzite phase nanostructures is also presented from a thermodynamic point of view, as shown in the phase diagram of Fig. 5. The solid lines denoted by $\mathrm{s}$ in Fig. 5 define the solidvapor equilibrium, while the dashed lines denoted by $\mathrm{m}$ give the equilibrium between the vapor phase and the metastable phase. It is noted that for a given temperature $T$, which is not equal to $T_{\text {tr }}$, the equilibrium vapor pressure $P_{m}$ of the metastable phase is always higher than the equilibrium vapor pressure $P_{s}$ of the stable crystal phase. Hence, the supersaturation $\Delta \mu_{s}=k T \ln \left(P / P_{s}\right)$ with respect to the stable phase will be higher than the supersaturation $\Delta \mu_{m}=k T \ln \left(P / P_{m}\right)$ with respect to the metastable phase, where $k$ is the Boltzmann 


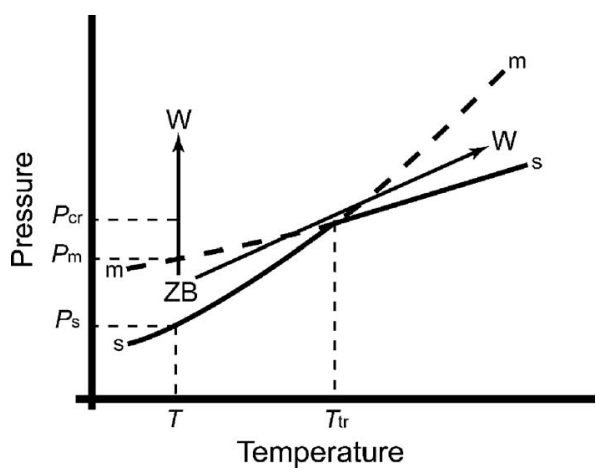

FIG. 5. Part of the phase diagram. The solid lines denoted by s define the solid-vapor equilibrium. The dashed lines denoted by $\mathrm{m}$ give the equilibrium between the vapor phase and the metastable phase. The arrowheads describe the final product.

constant and $P$ is the vapor pressure of the source material (i.e., $\mathrm{ZnSe}$ in our case). As a result, the stable phase is more likely to nucleate. In the present case, when the growth temperature increases from 650 to $700{ }^{\circ} \mathrm{C}$, the thermodynamically stable phase of $\mathrm{ZnSe}$ will change from zinc blende at low temperature to wurtzite structure at relatively high temperature as discussed previously; consequently, the wurtzite structures will nucleate. On the other hand, we should also note that the cubic products formed in the early $30 \mathrm{~min}$ will transform to wurtzite phase when the temperature is above $T_{\mathrm{tr}}$, and the defects, such as stacking faults and twin lamellae, will provide the nucleation sites. ${ }^{24}$ Hence, the product will change from zinc blende (ZB in Fig. 5) to wurtzite phase (W in Fig. 5). Together with our recently reported wurtzite nanorings grown at $650{ }^{\circ} \mathrm{C}$ and 0.2 torr, ${ }^{17}$ which can be attributed to the pressure effect described by Ostwald's step rule, a full picture of the formation of the wurtzite phase $\mathrm{ZnSe}$ can be summarized in the pressure-temperature phase diagram.

On the other hand, the surface energy of the outermost faces plays an important role for the stable structure of $\mathrm{ZnSe}$ at room temperature is zinc blende. ${ }^{13}$ Notably, our results show that the ZnSe 1D nanostructures synthesized at $90 \mathrm{~min}$ maintain wurtzite structure after the furnace is cooled down to room temperature. Here we will study the effects of outermost faces of the wurtzite $\mathrm{ZnSe}$ nanostructures on its stability. Based on the studies of $\mathrm{ZnS},{ }^{18}$ one can deduce that the nonpolar faces of (110) for zinc blende phase and (112 0 ) for wurtzite phase have the lowest surface energies of $0.39-0.65$ and $0.28-0.49 \mathrm{~J} \mathrm{~m}^{-2}$, respectively. Simulations indicate that for wurtzite phase, (0001) face has the highest surface energy of $0.91-1.52 \mathrm{~J} \mathrm{~m}^{-2}$, while for zinc blende phase, both (111) and (100) faces have the two highest surface energies of 1.84 and $2.56 \mathrm{~J} \mathrm{~m}^{-2}$, respectively. Since $\mathrm{ZnSe}$ and $\mathrm{ZnS}$ have nearly the same structures, the studies of the lowest surface energy faces of $\mathrm{ZnS}$ will be used to investigate the $\mathrm{ZnSe}$ nanostructures here, especially in consideration of polarity effect. Indeed, similar to the trend of $\mathrm{ZnS}$, the surface energies of (111) and (111) surfaces are higher than those of the
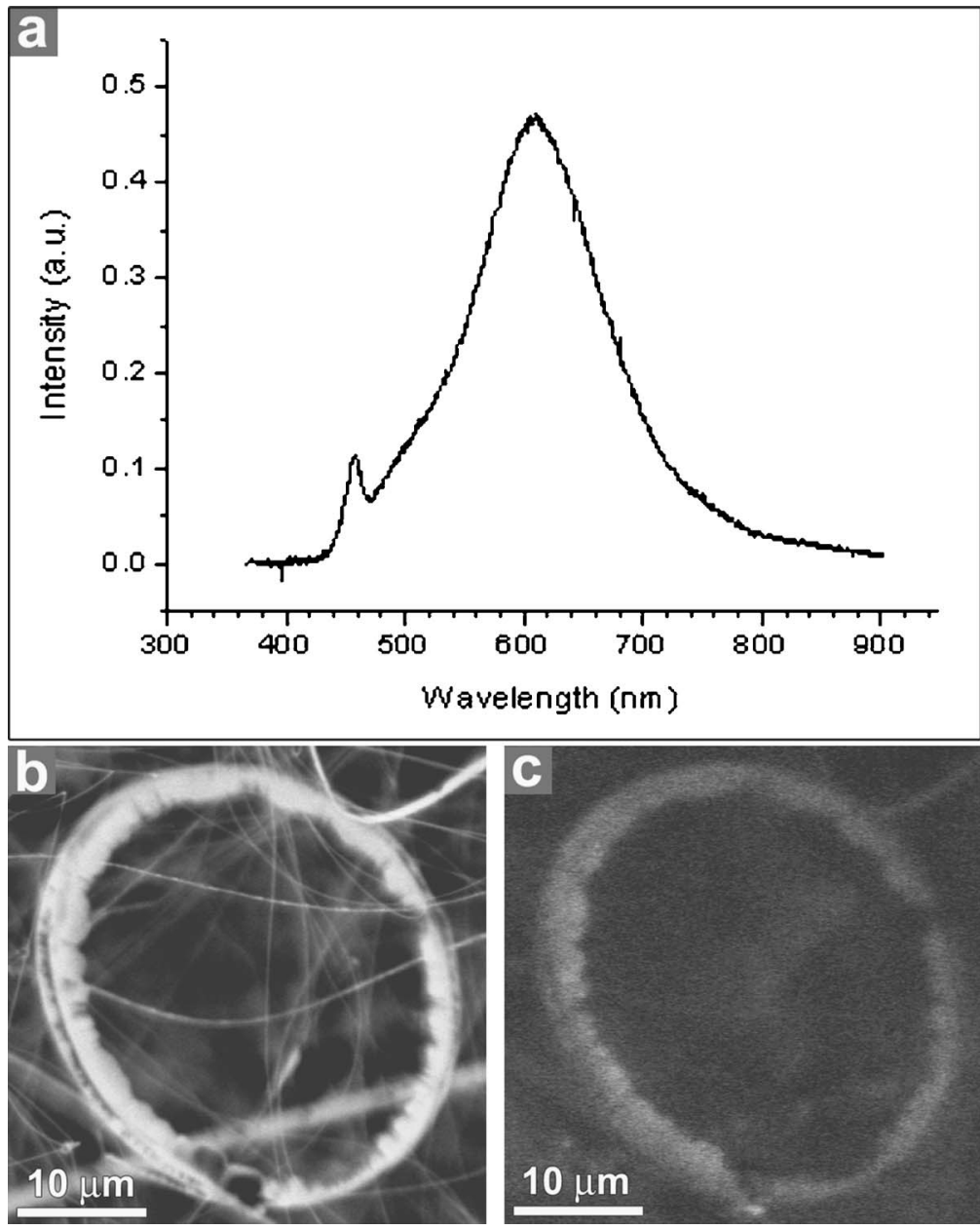

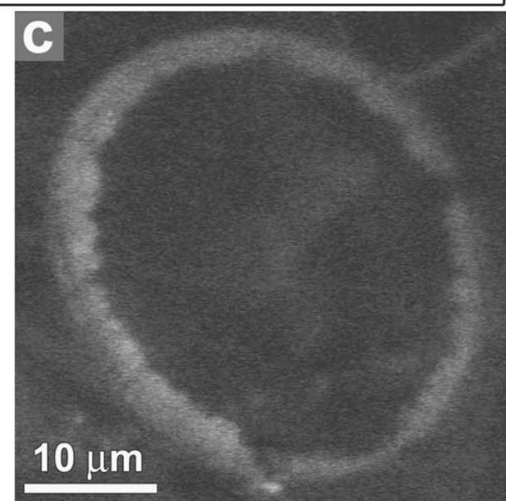

FIG. 6. (a) CL spectrum of the nanowheel showing the band transition emission and defect emission peaks. (b) SEM image of a typical nanowheel. (c) CL image of the nanowheel. 
(110) surfaces in zinc blende $\mathrm{ZnSe}$ if neglecting the Se-rich limit. ${ }^{25}$ For the wurtzite $\mathrm{ZnSe}$ nanostructures as shown in Fig. 2, the nanowheels have the dominant surfaces of $\pm\{2 \overline{1} \overline{1} 0\}$, which also have the lowest surface energies at ambient conditions and thus minimize the total surface energy of the nanostructures. As a result, the wurtzite $\mathrm{ZnSe}$ nanostructures can be obtained.

It is worthy to note that the discussion of the formation and stability of the wurtzite nanostructures here can be applied to discuss other nanostructures. For instance, we find that the low surface energy can also be applied to explain the results reported by Jiang et al. ${ }^{4}$

\section{Cathodoluminescence of nanostructures}

The SEM image of a ZnSe nanowheel and its CL image and spectrum at room temperature are shown in Fig. 6. The CL peak wavelength at $\sim 460 \mathrm{~nm} / \sim 2.7 \mathrm{eV}$ corresponds to the band edge transition energy $\left(E_{g}\right)$ of $\mathrm{ZnSe}$, while the band centered at $\sim 610 \mathrm{~nm} / \sim 2.03 \mathrm{eV}$ can be referred to the deep level emission. Since Au was used as the catalyst, it could potentially diffuse into the as-grown structures during the growth. Since the acceptor ionization energy of $\mathrm{Au}\left(E_{A}\right)$ is about $550 \mathrm{meV}$, the photon energy due to the transition between the conduction band and the Au acceptor can be estimated by $E=E_{g}-E_{A}{ }^{26}$ In this case, $E_{g}$ is $2.7 \mathrm{eV}$; thus the calculated $E$ is $2.15 \mathrm{eV}$, which is consistent with the result in Ref. 27. On the other hand, an excess of $\mathrm{Zn}$ in the $\mathrm{ZnSe}$ was reported to result in the emission at $2.03 \mathrm{eV},{ }^{28}$ while the dislocations, stacking faults, and nonstoichiometric defects would result in the emission at $2.2 \mathrm{eV}$, which may be another origins of the deep level emission peak. ${ }^{29}$ In our wurtzite $\mathrm{ZnSe}$ nanostructures, mismatch dislocations and stacking faults are obtained, as shown in the HRTEM images of Fig. 2(d). As a result, these low energy transitions due to unexpected $\mathrm{Au}$ dopant, excess of $\mathrm{Zn}$, and defects in the crystal structure can contribute to the low energy and broad emission band with center at $610 \mathrm{~nm}$.

\section{CONCLUSIONS}

In summary, the abnormal nanostructures of wurtzite nanowheels with teethlike extended patterns have been successfully synthesized in this study. HRTEM analysis implies that the wurtzite $\mathrm{ZnSe}$ nanostructures of the sample are obtained at the growth temperature of $\sim 700{ }^{\circ} \mathrm{C}$ and reactor pressure of 100 torr. By reducing the growth temperature to $\sim 650{ }^{\circ} \mathrm{C}$, zinc blende $\mathrm{ZnSe}$ nanostructures form. While there are a few reports in the formation mechanism and stability of the metastable wurtzite phase $\mathrm{ZnSe}$ nanostructures, the formation of the metastable wurtzite nanostructures has been proposed and the stability of the wurtzite nanostructures has also studied and explained by the surface energy of the nanostructures. The result showed that the wurtzite nanostructures had the dominant top/bottom surfaces of $\pm(2 \overline{1} \overline{1} 0)$ faces which had the lowest surface energies. Furthermore, the growth mechanism of the nanowheels has also been proposed using a combination of VS mechanism and the selfcatalyzed growth process in two stages. The first stage is the formation of the nanowheels base via VS mechanism. In the second step, the $\mathrm{ZnSe}$ vapor continuously nucleates and grows along the [0001] direction and finally forms the teethlike extended patterns through the self-catalyst growth process. The CL of the ZnSe nanowheels has been studied. The spectrum shows the band edge emission of the ZnSe nanostructures and the broad emission at lower photon energy.

\section{ACKNOWLEDGMENTS}

This work was supported by the University Development Fund (UDF) and the seed funding of the University of Hong Kong. One of the authors (T.I.Y.) thanks the support of seed funding from the University of Hong Kong. The authors thank the discussion with I. Markov supported by William Mong Visiting Fellowship of the University of Hong Kong.

${ }^{1}$ D. M. Bagnall, Y. F. Chen, T. Yao, S. Koyama, M. Y. Shen, and T. Goto, Appl. Phys. Lett. 70, 2230 (1997); T. Matasuoka, Adv. Mater. (Weinheim, Ger.) 8, 469 (1996).

${ }^{2}$ P. V. Kamat, Chem. Rev. (Washington, D.C.) 93, 267 (1993).

${ }^{3}$ H. W. Schock, Sol. Energy Mater. Sol. Cells 34, 19 (1994).

${ }^{4}$ Y. Jiang, X. M. Meng, W. C. Yiu, J. Liu, J. X. Ding, C. S. Lee, and S. T. Lee, J. Phys. Chem. B 108, 2784 (2004).

${ }^{5}$ J. H. Choy, E. S. Jang, J. H. Won, J. H. Chung, D. J. Jang, and Y. W. Kim, Adv. Mater. (Weinheim, Ger.) 15, 1911 (2003); Z. W. Pan, Z. R. Dai, and Z. L. Wang, Science 291, 1947 (2001).

${ }^{6}$ X. Y. Kong and Z. L. Wang, Nano Lett. 3, 1625 (2003); C. Ma, D. Moore, J. Li, and Z. L. Wang, Adv. Mater. (Weinheim, Ger.) 15, 228 (2003).

${ }^{7}$ A. B. Djurišić et al., Adv. Funct. Mater. 14, 856 (2004).

${ }^{8}$ Y. Jiang, W. J. Zhang, J. S. Jie, X. M. Meng, and J. A. Zapien, Adv. Mater. (Weinheim, Ger.) 18, 1527 (2006).

${ }^{9}$ R. Rujkorakarn and A. J. Nelson, J. Appl. Phys. 87, 8557 (2000).

${ }^{10}$ R. Pässler et al., J. Appl. Phys. 86, 4403 (1999).

${ }^{11}$ A. Slobodskyy, C. Gould, T. Slobodskyy, C. R. Becker, G. Schmidt, and L. W. Molenkamp, Phys. Rev. Lett. 90, 246601 (2003).

${ }^{12}$ J. F. Holzman, F. E. Vermeulen, S. E. Irvine, and A. Y. Elezzabi, Appl. Phys. Lett. 81, 2294 (2002).

${ }^{13}$ C. Y. Yeh, Z. W. Lu, S. Froyen, and A. Zunger, Phys. Rev. B 46, 10086 (1992).

${ }^{14}$ S. L. Xiong, J. M. Shen, Q. Xie, Y. Q. Gao, Q. Tang, and Y. T. Qian, Adv. Funct. Mater. 15, 1787 (2005); W. C. H. Choy, C. F. Guo, Y. P. Leung, G. Z. Wang, and T. I. Yuk, Appl. Phys. A: Mater. Sci. Process. 83, 301 (2006).

${ }^{15}$ J. Q. Hu, Y. Bando, and D. Golberg, Small 1, 95 (2005); S. Y. Liu, W. C. H. Choy, L. Jin, Y. P. Leung, G. P. Zheng, J. Wang, and A. K. Soh, J. Phys. Chem. C. 111, 9055 (2007).

${ }^{16}$ Z. L. Wang, X. Y. Kong, and J. M. Zuo, Phys. Rev. Lett. 91, 185502 (2003).

${ }^{17}$ Y. P. Leung, W. C. H. Choy, I. Markov, G. K. H. Pang, H. C. Ong, and T. I. Yuk, Appl. Phys. Lett. 88, 183110 (2006).

${ }^{18}$ Z. W. Wang, L. L. Daemen, Y. S. Zhao, C. S. Zha, R. T. Downs, X. D. Wang, Z. L. Wang, and R. J. Hemley, Nat. Mater. 4, 922 (2005).

${ }^{19}$ R. S. Wagner and W. C. Ellis, Appl. Phys. Lett. 4, 89 (1964).

${ }^{20}$ H. Okada, T. Kawanaka, and S. Ohmoto, J. Cryst. Growth 165, 31 (1996).

${ }^{21}$ S. B. Qadri, E. F. Skelton, D. Hsu, A. D. Dinsmore, J. Yang, H. F. Gray, and B. R. Ratna, Phys. Rev. B 60, 9191 (1999).

${ }^{22}$ I. V. Markov, Crystal Growth for Beginners, 2nd ed. (World Scientific, Singapore, 2003), Chap. 2.

${ }^{23}$ Y. Ding, X. D. Wang, and Z. L. Wang, Chem. Phys. Lett. 398, 32 (2004).

${ }^{24}$ X. T. Zhang, K. M. Ip, Z. Liu, Y. P. Leung, Q. Li, and S. K. Hark, Appl. Phys. Lett. 84, 2641 (2004).

${ }^{25}$ S. B. Zhang and S. H. Wei, Phys. Rev. Lett. 92, 086102 (2004).

${ }^{26}$ B. Xiang et al., Appl. Phys. Lett. 82, 3330 (2003).

${ }^{27}$ P. J. Dean, B. J. Fitzpatrick, and R. N. Bhargava, Phys. Rev. B 26, 2016 (1982).

${ }^{28}$ V. V. Blinov, E. M. Gavrishchuk, V. G. Galstyan, V. S. Zimogorshii, I. A. Karetnikov, N. K. Morozova, and V. G. Plotnichenko, Inorg. Mater. 37, $1228(2001)$.

${ }^{29}$ X. T. Zhang, Z. Liu, Y. P. Leung, Q. Li, and S. K. Hark, Appl. Phys. Lett. 83, 5533 (2003). 